\title{
Methane Gas Sensor Based on Microstructured Highly Sensitive Hybrid Porous Core Photonic Crystal Fiber
}

\author{
Md. Ranju Sardar, Mohammad Faisal \\ Department of Electrical \& Electronic Engineering (EEE), Bangladesh University of Engineering \& Technology (BUET) \\ Dhaka-1000, Bangladesh \\ Email: ranju_sardar@yahoo.com, mdfaisal@eee.buet.ac.bd
}

How to cite this paper: Sardar, M.R. and Faisal, M. (2019) Methane Gas Sensor Based on Microstructured Highly Sensitive Hybrid Porous Core Photonic Crystal Fiber. Journal of Sensor Technology, 9, 12-26. https://doi.org/10.4236/jst.2019.91002

Received: February 6, 2019

Accepted: March 15, 2019

Published: March 18, 2019

Copyright (๑) 2019 by author(s) and Scientific Research Publishing Inc. This work is licensed under the Creative Commons Attribution International License (CC BY 4.0).

http://creativecommons.org/licenses/by/4.0/ cc) (i) Open Access

\begin{abstract}
We have demonstrated and analyzed the methane gas sensor based on octagonal cladding and hexagonal hybrid porous core photonic crystal fiber (HPC-PCF) for gas detection purpose. The proposed design of HPC-PCF has been numerically investigated by COMSOL Multiphysics software through utilizing the full vectorial finite element method (FEM). The optical characteristics of HPC-PCF as well as confinement loss, relative sensitivity and refractive index, effective area, nonlinearity and numerical aperture are optimized properly by changing the geometrical parameters as well as air filling ratio, air hole diameter, pitch constant of cladding and porosity of the core. In this simulation work, we have achieved optimum relative sensitivity of $21.2 \%$, and confinement loss of $0.000025 \mathrm{~dB} / \mathrm{m}$ at $3 \mu \mathrm{m}$ pitch, 0.7 air filling ratio of the cladding and $29 \%$ porosity of the core for $3.5 \mu \mathrm{m}$ absorption wavelength of $\mathrm{CH}_{4}$ gas. This proposed design of HPC-PCF will keep exclusive contribution for detecting the $\mathrm{CH}_{4}$ gas accurately.
\end{abstract}

\section{Keywords}

HPC-PCF, Relative Sensitivity, Confinement Loss, Porosity, $\mathrm{CH}_{4}$ Gas and Gas Sensor

\section{Introduction}

Conventional optical fiber technology provides greater bandwidth services and excellent data accuracy than copper wire in telecommunication systems. An application of optical fiber is fixed due to its geometrical restrictions and it is typically used in telecommunication systems. But we can overcome the application 
restrictions of optical fiber through re-structuring the geometrical model. One of the re-structuring models of optical fiber is called the photonic crystal fiber (PCF). The PCF having microstructured array air holes regularly spreads across the whole length of the fiber [1] [2]. Our proposed design is referred to as hybrid porous core photonic crystal fiber (HPC-PCF) where solid core of PCF is tailored into porous core through creating rectangular and circular air holes in the core area [3] [4]. The HPC-PCF confines the optical signal in the core by utilizing the modified total internal reflection (MTIR) of light just like solid core PCF or by utilizing photonic bandgaps (PBG) effect just like hollow core PCF. The refractive index of the core and the refractive index of the cladding of HPC-PCF is a comparable matter. When the refractive index of the core is large with respect to cladding, the HPC-PCF confines the optical signal in the core by utilizing MTIR technique [5]. On the other hand, when the refractive index of the core is low with respect to cladding, HPC-PCF confines the optical signal in the core by utilizing PBG effects [6] [7] where length of wavelength is greater than silica gap between two air holes of cladding. Recently, the HPC-PCF has been used for various sensors like gas sensor [8] [9], liquid sensor [10] [11], humidity and temperature sensor [12], air pollution monitoring sensor [13] and so on. In addition, the HPC-PCF is not only used in sensing purposes but also uses in various biological diagnosis like skin cancer [14], breast cancer [15], colon cancer [16], DNA [17], RNA [18], proteins [19] and frequency time domain spectroscope [18] etc. The background material of our proposed design of HPC-PCF is purely silica material and in these design air holes of the core is filled with $\mathrm{CH}_{4}$ gas. For the purely silica material, interaction happens between optical signal and $\mathrm{CH}_{4}$ gas at $3.5 \mu \mathrm{m}$ absorption wavelength of $\mathrm{CH}_{4}$ gas. We can use various polymer materials as background materials instead of silica material like Cyclo-Olefin Polymer (COP), polyethylene, polymethyl-methacrylate (PMMA), Cyclic Olefin Copolymer (COC), Teflon and so on. In that case, HPC-PCF will operate in THz frequencies. In fact, the HPC-PCF have many properties like relative sensitivity [20] [21], low confinement loss [22] [23], birefringence [24], dispersion [25] [26], nonlinearity [27] and etc. The various shapes of HPC-PCF have supremacy in optical properties. The most familiar shape of HPC-PCF are available such as decagonal [28], octagonal [29], hexagonal, elliptical [30], circular honey comb cladding [31] and so on. The HPC-PCF is optimized by changing the geometrical parameters like air hole diameter, air filling fractions, pitch constant and porosity of the core. In this design, $3 \mu \mathrm{m}$ pitches, 0.7 air filling ratio of cladding and $29 \%$ porosity of the core are chosen as optimum value, since in these values, we have obtained the highest relative sensitivity of $21.2 \%$, and minimum confinement loss of $0.000025 \mathrm{~dB} / \mathrm{m}$ at $3.5 \mu \mathrm{m}$ absorption wavelength of $\mathrm{CH}_{4}$ gas than other values. The HPC-PCF can be used successfully as a sensing device through accomplishing the whole fabrication process properly. Most well-known fabrication processes of PCF are 3D-printing, sol-gel, capillary stacking, stack and drilling, extrusion and slurry casting. Among them, extrusion 
is the most suitable fabrication process for HPC-PCF design. The prime dream of proposed design of HPC-PCF is to improve relative sensitivity and decrease confinement loss at minimum level that will sense $\mathrm{CH}_{4}$ gas accurately.

\section{Experimental Set up and Sensing Mechanism of Proposed HPC-PCF}

Figure 1 demonstrates the proposed schematic experimental arrangement of sensing the $\mathrm{CH}_{4}$ gas through utilizing the HPC-PCF. The $\mathrm{CH}_{4}$ gas sensing system is made up of miscellaneous elements as well as vacuum pump, gas cylinder, gas valve, laser source, optical spectrum analyzer (OSA) and chamber unit. It is seen from Figure 1 that the chamber unit is connected with laser source, OSA, gas cylinder through gas valve and the vacuum pump. In this system, the laser source is used for supplying the input optical signal into the chamber unit and OSA is used for detecting the output optical signal. On the other hand, gas valve is to control the gas flow rate into the chamber unit at the precise pressure and vacuum pump is used to collect the gas from the chamber unit at the reasonable temperature. The $\mathrm{CH}_{4}$ gas sensing mechanism of HPC-PCF is that an interaction occurs between $\mathrm{CH}_{4}$ gas and optical signal at the $3.5 \mu \mathrm{m}$ absorption wavelength peak of $\mathrm{CH}_{4}$ gas into the chamber unit. In that case, gas constituents absorb the energy of photon of optical signal and the intensity of optical signal varies with respect to absence of gas. This whole matter is clearly explained by Beer Lambert Law (BLL) in the later part of this manuscript.

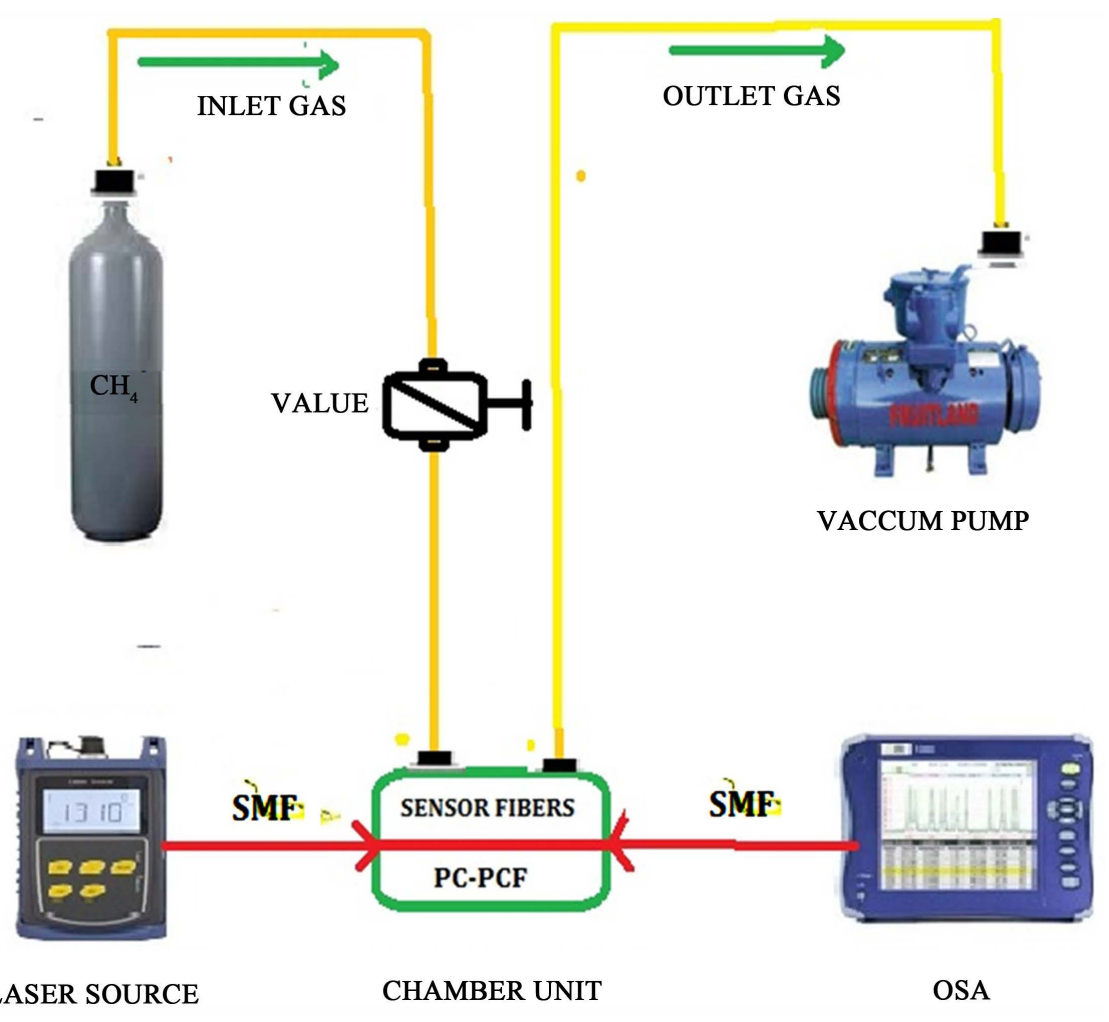

Figure 1. An experimental set up for sensing $\mathrm{CH}_{4}$ gas of proposed HPC-PCF. 


\section{Geometrical Configuration of HPC-PCF}

Figure 2(a) demonstrates the whole cross sectional view of the proposed design of HPC-PCF. The entire design of HPC-PCF is composed of three parts such as core, cladding and the perfectly match layer (PML). In this design, the perfectly match layer (PML) is made by purely silica material and thickness of the perfectly match layer is $1 \mu \mathrm{m}$. The PML does the function of an absorber for the optical signal of the HPC-PCF so that no reflection occurs at the boundary. The remaining parts of the proposed design of HPC-PCF are cladding and core which can be shown by Figure 2(b) and Figure 2(c), respectively. Figure 2(b) illustrates the cross sectional view of the circular air hole based porous cladding. The design parameters of the cladding are silica material, pitch and the circular air holes. Two circular rings are available in cladding where inner ring contain 16 air holes and outer ring contains 24 air holes. Figure 2(c) exhibits the cross sectional view of core of the HPC-PCF. In this design, the core contains five circular air holes and eight rectangular slots. The purpose of using different shape of air hole in core area is to regulate the porosity of the core separately and optimize it for the better relative sensitivity and low confinement loss. In this design, six porosities of the core like 22\%, 24\%, 25\%, 26\%, 28\% and $29 \%$ are used for optimizing the HPC-PCF. In this design, methane gas is utilized in air holes of the core having refractive index of 1.000444 [32].

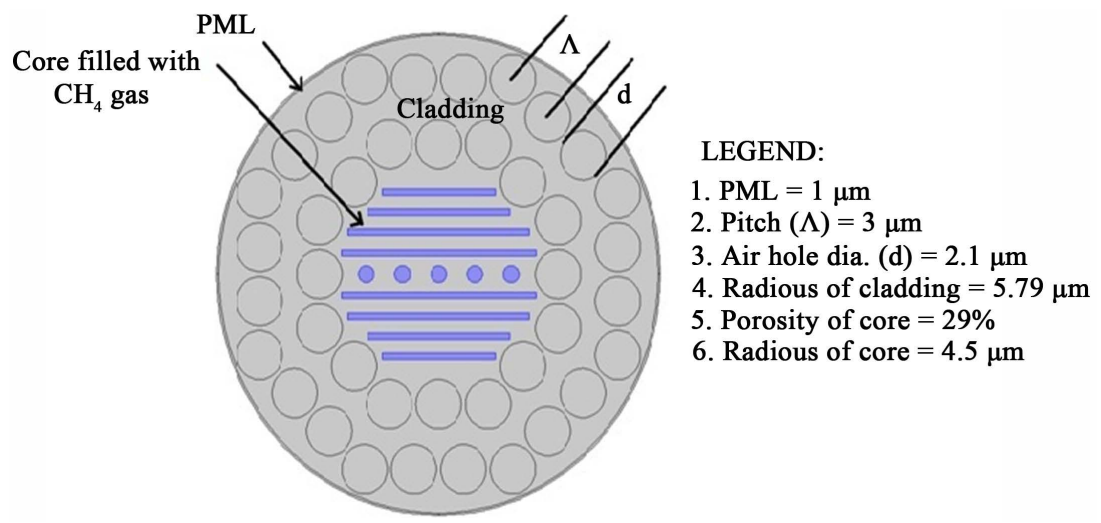

(a)

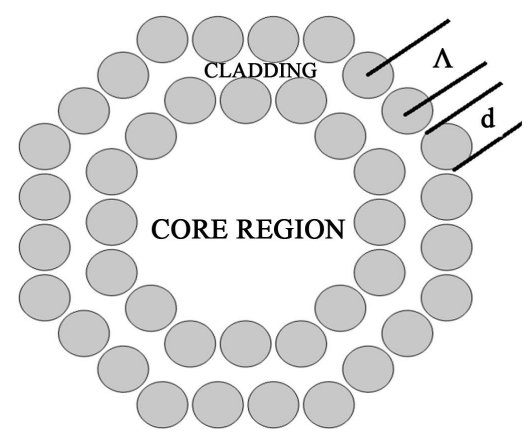

(b)

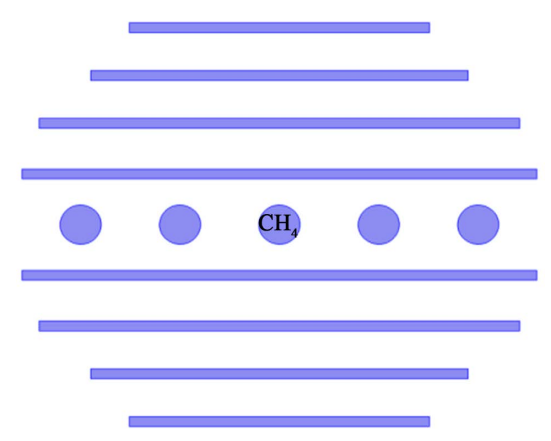

(c)

Figure 2. (a) An entire cross section view of HPC-PCF; (b) Cross section view of cladding of HPC-PCF; (c) Cross section view of core of HPC-PCF. 


\section{Signal Confinement Technique of HPC-PCF}

Figure 3 represents the signal confinement view of HPC-PCF at $3.5 \mu \mathrm{m}$ operating wavelength of $\mathrm{CH}_{4}$ gas. The HPC-PCF shows feature like hollow core PCF and confines the optical signal into the core by utilizing the mechanism of PBG effects. This phenomenon is clearly described in the later section of this manuscript.

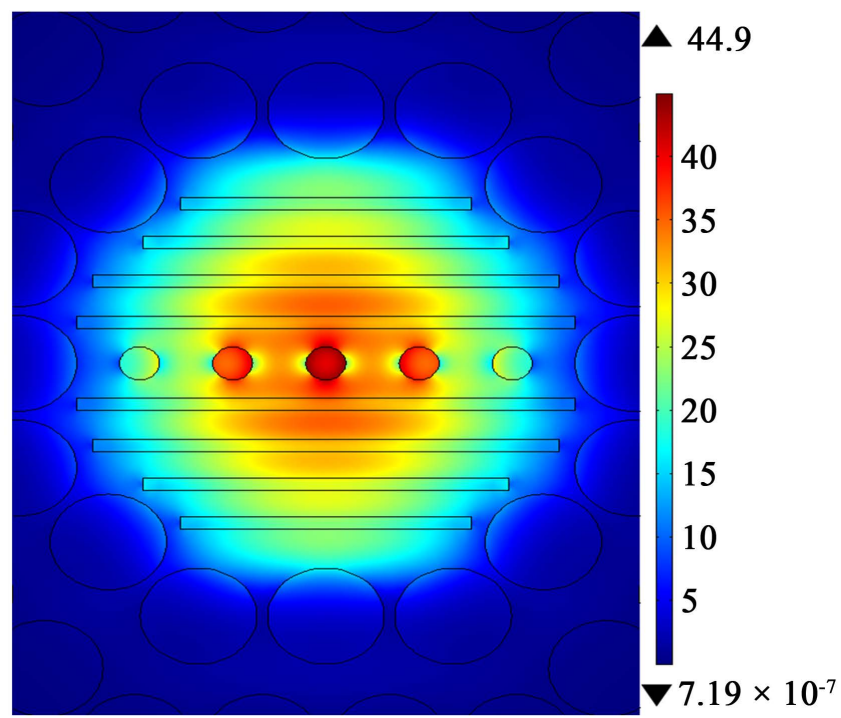

Figure 3. Signal confinement view of HPC-PCF at $3.5 \mu \mathrm{m}$ wavelength.

\section{Equations}

The confinement loss is the significant optical characteristic of HPC-PCF. It is dominated by air hole shape, number, background material, thickness of perfectly match layer (PML), wavelength and etc. When optical signal penetrates into core and then goes in cladding region, this matter is known as confinement loss. The confinement loss of HPC-PCF can be determined by following equation

$$
L_{c}=8.686 k_{0} I_{m}\left[n_{\text {eff }}\right]
$$

where $k_{0}$ is known as wave number for free-space and $I_{m}\left[n_{\text {eff }}\right]$ is known as imaginary part of the effective mode index. The relative sensitivity means that the interaction between the light and gas components at the precise wavelength of optical signal. Relative sensitivity is integrated with absorption coefficient of the gas of Beer-Lambert law. The Beer Lambert law can be expressed by the following equation

$$
I(\lambda)=I_{0}(\lambda) \exp \left(-r \theta_{m} l_{c}\right)
$$

where $I(\lambda)$ and $I_{0}(\lambda)$ are known as amplitudes of optical signal with and without gas, $r$ is known as relative sensitivity, $a_{m}$ known as absorption coefficient, $l_{e}$ is known as length of channel and is known as absorption wavelength of $\mathrm{CH}_{4}$ gas. The relative sensitivity of HPC-PCF can be expressed by the following equation 


$$
r=\frac{n_{r}}{n_{e f f}}
$$

where $n_{r}$ is identified as refractive index and $n_{\text {eff }}$ is identified as effective refractive index. The coefficient $f$ is defined as the optical power available in air holes to total optical power of the HPC-PCF which can be mathematically expressed by the following equation

$$
f=\frac{\int_{\text {Hole }}\left(E_{x} H_{y}-E_{y} H_{x}\right) \mathrm{d} x \mathrm{~d} y}{\int_{\text {Total }}\left(E_{x} H_{y}-E_{y} H_{x}\right) \mathrm{d} x \mathrm{~d} y}
$$

where $E_{x}, E_{y}$, are known as transverse electric field and $H_{y}, H_{\mathrm{y}}$ are known as longitudinal magnetic field respectively. The refractive index is the ratio of length of wavelength of optical signal in the air to the length of wavelength of optical signal in a medium. Mathematically, it can be expressed by the following equation

$$
n=\frac{\lambda_{0}}{\lambda}
$$

where $n$ is the refractive index, $\lambda_{0}$ is the length of wavelength in air and $\lambda$ is the length of wavelength in a medium. The porosity is the geometric property of the HPC-PCF. Typically, it is the ratio of the total air hole area of the core and to the total cross sectional area of the core which can be expressed by the following equation

$$
P_{s}=\frac{a}{A} \times 100 \%
$$

where $a$ is the total cross section area of air holes into core area and $A$ is total cross section area of the core respectively. The area which is used by optical signal in the core while propagation in waveguide is known as effective area of HPC-PCF. The effective area of the core of HPC-PCF can be expressed by the following equation

$$
A_{e f f}=\frac{\left[\int F(x) x \mathrm{~d} x\right]^{2}}{\left[\int F^{2}(x) \mathrm{d} x\right]^{2}}
$$

where $A_{\text {eff }}$ is identified as effective area of the core and $F(x)=\left|E_{x}\right|^{2}$ is identified as transverse electric field distribution in the core area of HPC-PCF. Nonlinearity is an important feature of HPC-PCF which is inversely proportional to effective area of the core. The nonlinearity of HPC-PCF can be demonstrated by the following equation

$$
N L=\frac{2 \pi n_{2}}{\lambda A_{e f f}}
$$

where $N L$ is known as nonlinearity, $n_{2}$ is known as refractive index, $\lambda$ is known as length of wavelength and $A_{\text {eff }}$ is known as effective area of the core of HPC-PCF. The higher value of numerical aperture is more important to operate HPC-PCF as a sensor and it can be achieved effortlessly when the refractive index difference between the core and the cladding is large. The numerical aperture 
of HPC-PCF can be determined by the following equation

$$
N A=\frac{1}{\sqrt{1+\frac{\pi A_{\text {eff }} f^{2}}{c^{2}}}}
$$

where $f$ is referred to as frequency of optical signal, $c$ is referred to as velocity of optical signal and $A_{\text {eff }}$ is referred to as effective area of the core of HPC-PCF.

\section{Simulations}

Figure 4 shows the variations of confinement loss with respect to different wavelengths at different pitches of the cladding of HPC-PCF. In Figure 4, four pitches of cladding like $2.80 \mu \mathrm{m}, 2.85 \mu \mathrm{m}, 2.90 \mu \mathrm{m}$ and $3.0 \mu \mathrm{m}$ are regulated for observing the variations of confinement loss corresponding to each value of pitch. It is seen from Figure 4 that minimum pitch of the cladding provides maximum confinement loss with respect to maximum pitch. This phenomenon is realistic because minimum pitch of cladding produces minimum refractive index difference between core and cladding. For this reason, more optical signal to penetrate into core and goes in the cladding area and finally causes maximum confinement loss. It is also seen from Figure 4 that the confinement loss increases with increasing wavelength because higher wavelength creates lower refractive index difference between core and the cladding area. As a result, maximum optical signal infiltrates in cladding from the core. So higher wavelength offers higher confinement loss than that of lower wavelength. Figure 5 explains the fluctuations of relative sensitivity with respect to diverse wavelengths at diverse pitches of cladding of HPC-PCF. In Figure 5, four pitches of the cladding such as $2.80 \mu \mathrm{m}, 2.85 \mu \mathrm{m}, 2.90 \mu \mathrm{m}$ and $3.0 \mu \mathrm{m}$ are also regulated for watching the variations of relative sensitivity. In Figure 5, higher pitch of cladding offers higher relative sensitivity than that of the lower pitch. This statement is true because higher pitch of the cladding produces higher refractive index difference between core and the cladding region. In that case, minimum optical signal leaks

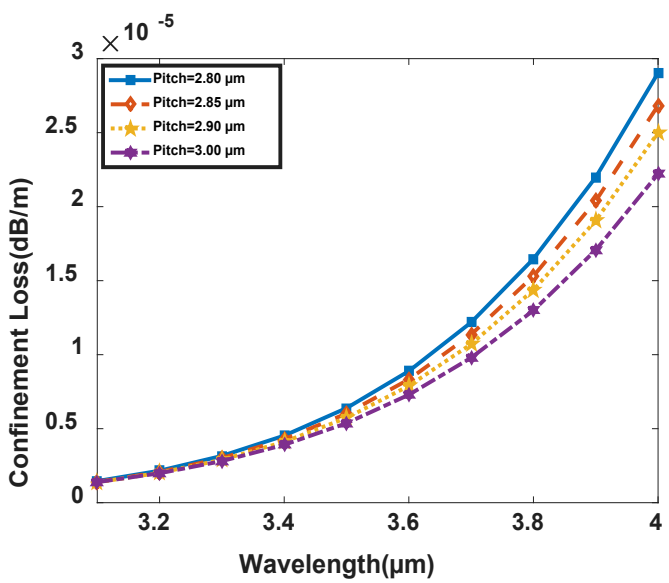

Figure 4. Confinement loss vs. wavelength curve for various pitches of cladding of HPC-PCF. 


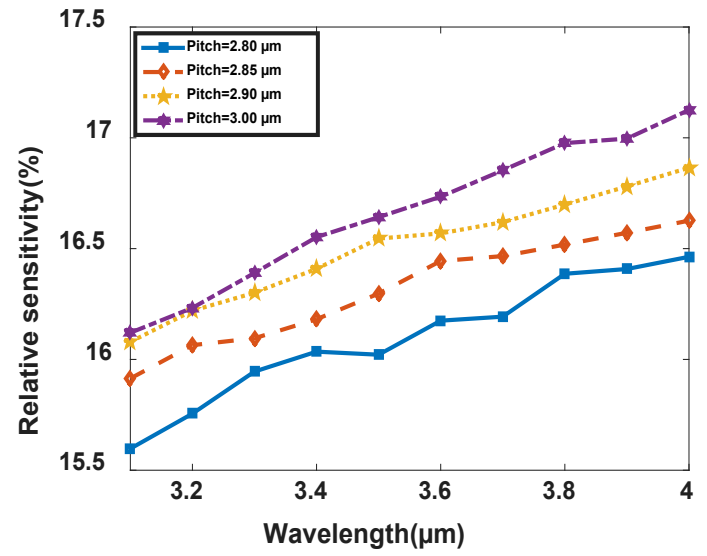

Figure 5. Relative sensitive vs. wavelength curve for various pitches of cladding of HPC-PCF.

from the core, more interaction occurs in core between optical signal and gas elements and this causes higher relative sensitivity.

Figure 6 demonstrates the wavelength versus confinement loss curve at various porosities of core of HPC-PCF Figure 6, four porosities of core like 22\%, $24 \%, 25 \%$ and $26 \%$ are regulated for testing the fluctuations of confinement loss. It is realized from Figure 6 that minimum porosity of the core gives the minimum confinement loss than that of the maximum porosity. This statement is valid because minimum porosity of the core creates maximum refractive index difference between core and the cladding than the maximum porosity. In that case, minimum optical signal penetrates from core. For this reason, minimum porosity of the core provides minimum confinement loss than the maximum porosity. Figure 7 exhibits the wavelength versus relative sensitivity curve at miscellaneous porosities of the core of HPC-PCF. In Figure 7, 22\%, 24\%, 25\% and $26 \%$ porosities of the core are assumed for checking the deviations of relative sensitivity. It is observed from Figure 7 that maximum porosity of the core provides maximum relative sensitivity than the minimum porosity. This statement is also valid because maximum porosity of the core contains maximum amount of gas in the core area. In that case, higher interaction happens into the core between light and the gas ingredients and displays the higher relative sensitivity.

The characteristics of effective refractive index as a function of wavelength at diverse porosities of the core of HPC-PCF are shown in Figure 8. In Figure 8, diverse porosities of the core of HPC-PCF give the diverse effective refractive indices. It is seen from Figure 8 that minimum porosity of the core provides maximum effective refractive index with respect to maximum porosity. This comment is logical because minimum porosity of the core produces denser medium. Due to effect of denser medium, the value of signal velocity in medium reduces and effective refractive index increases eventually. It is also seen from Figure 8 that effective refractive index decreases with increasing wavelength because at higher wavelength, the velocity of optical signal is higher and corresponding refractive index is lower. 


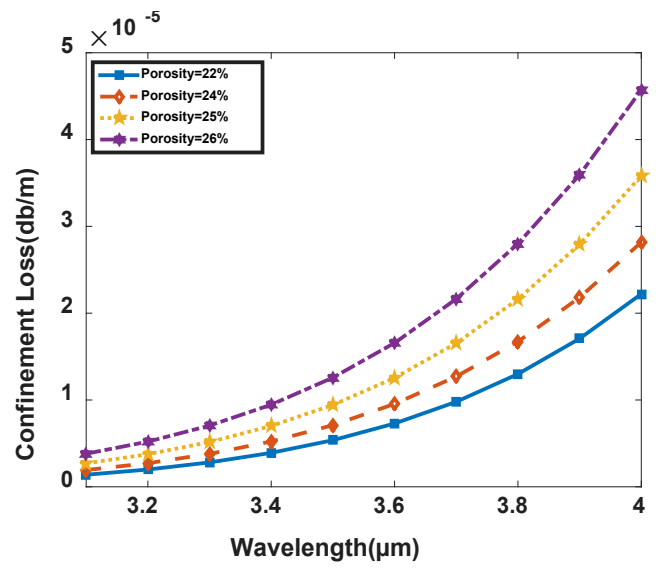

Figure 6. Confinement loss vs. wavelength curve for various porosities of the core of HPC-PCF.

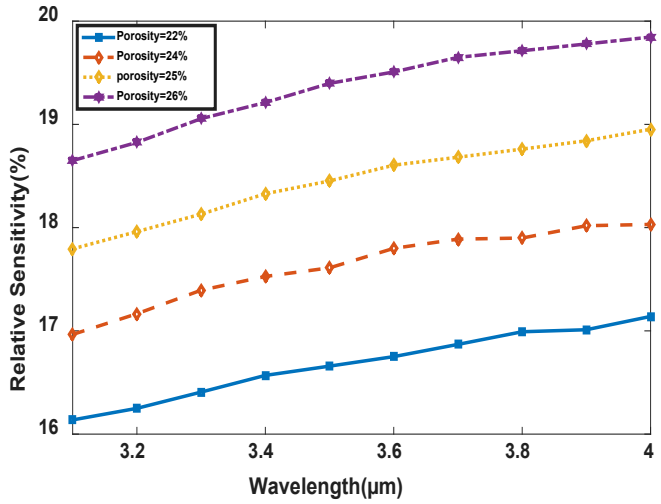

Figure 7. Relative sensitivity as a function of wavelength at different porosities of the core of HPC-PCF.

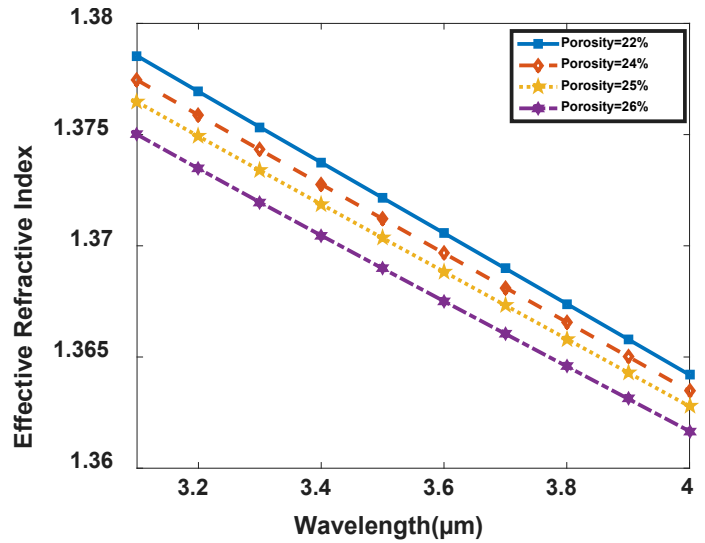

Figure 8. An effective refractive index as a function of wavelength at diverse porosities of the core of HPC-PCF.

Figure 9 shows the variations of effective area of the core of HPC-PCF corresponding to various pitches of the cladding. In Figure 9, $2.80 \mu \mathrm{m}, 2.85 \mu \mathrm{m}$, $2.90 \mu \mathrm{m}$ and $3.0 \mu \mathrm{m}$ pitch of the cladding are considered for testing the variations of effective area of the core. It is observed from Figure 9 that minimum 


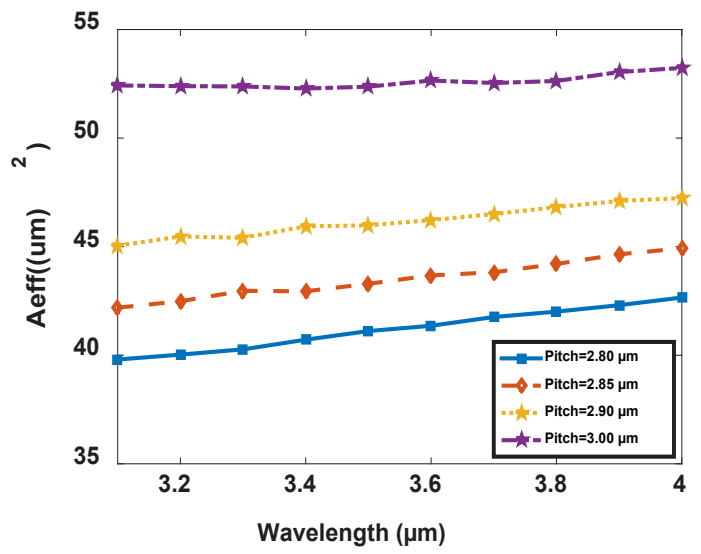

Figure 9. An effective area as a function of wavelength at diverse pitches of the cladding of HPC-PCF.

pitch of the cladding provides minimum effective area than maximum pitch. This statement is true because geometrically when the pitch of the cladding decreases, area of the core decreases automatically. On the other hand, when the pitch of the cladding increases, area of the core also increases. Note that the effective area is the major part of the total core area. So the lower pitch of the cladding provides the lower effective area than the higher pitch.

Figure 10 expresses the fluctuations of nonlinearity with respect to wavelength at different pitches of the cladding of HPC-PCF. It is seen from Figure 10 that minimum pitch of the cladding offers maximum nonlinearity than the maximum pitch. This result is true because in Figure 9, we have seen that minimum pitch of the cladding provides minimum effective area of the core than that of maximum pitch. In addition, nonlinearity is inversely proportional to effective area of the core and it is given by "Equation (8)". For this reason, minimum pitch of the cladding provides the maximum nonlinearity than that of the maximum pitch. Figure 11 displays the numerical aperture versus wavelength curve at different pitches of the cladding of HPC-PCF. In Figure 11, different pitches of the cladding including $2.80 \mu \mathrm{m}, 2.85 \mu \mathrm{m}, 2.90 \mu \mathrm{m}$ and $3.0 \mu \mathrm{m}$ are regulated for examining the switching value of the numerical aperture. It is seen from Figure 11 that numerical aperture increases with increasing wavelength because when wavelength increases, frequency automatically decreases and numerical aperture correspondingly increases. Note that frequency is inversely proportional to numerical aperture and it given by "Equation (9)". It is also observed from Figure 11 that minimum pitch of the cladding displays the maximum numerical aperture than the maximum pitch. This phenomena is also true because numerical aperture is inversely proportional to effective area of the core and it is given by "Equation (9)". Again it is seen from Figure 9 that minimum pitch of the cladding gives the minimum effective area of the core than the maximum pitch. So minimum pitch provides the maximum numerical aperture than the maximum pitch of the cladding.

In Figure 12, 22\%, 24\%, 25\%,26\%, 28\% and $29 \%$ porosities of the core are 


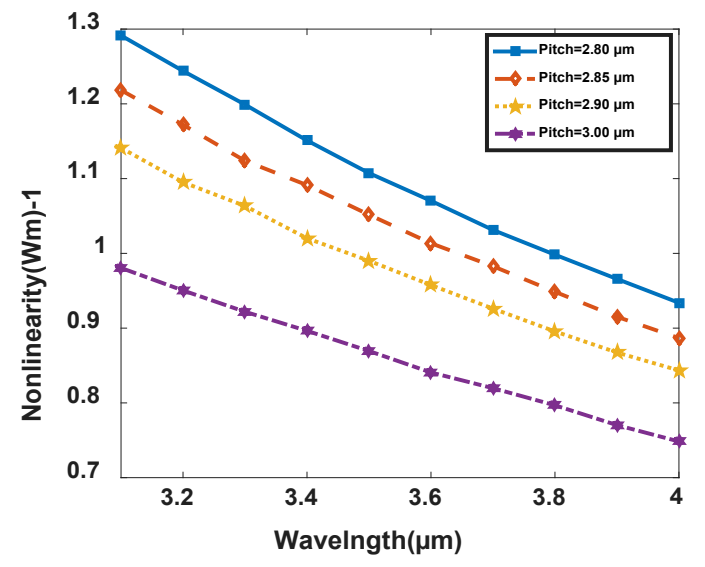

Figure 10. Nonlinearity as a function of wavelength at diverse pitches of the cladding of HPC-PCF.

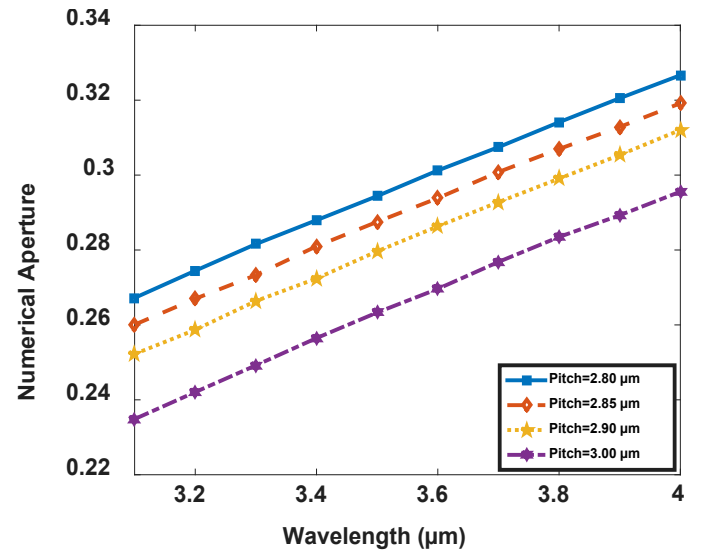

Figure 11. Numerical aperture as a function of wavelength at diverse pitches of the cladding of HPC-PCF.

considered for observing the variations of confinement loss with respect to each porosity value for the $3.5 \mu \mathrm{m}$ absorption wavelength of $\mathrm{CH}_{4}$ gas at $3.0 \mu \mathrm{m}$ pitch and 0.7 air filling fraction of the cladding. It is seen from Figure 12 that minimum porosity of $22 \%$ provides minimum confinement loss of $0.000005 \mathrm{~dB} / \mathrm{m}$ and maximum porosity of $29 \%$ provides of $0.000025 \mathrm{~dB} / \mathrm{m}$ for the $3.5 \mu \mathrm{m}$ absorption wavelength of $\mathrm{CH}_{4}$ gas. This result is reasonable because minimum porosity of $22 \%$ of the core creates maximum refractive index difference between core and the cladding than the maximum porosity of $29 \%$. In that case, minimum optical signal penetrates from the core and goes in cladding region that happens minimum confinement loss. In Figure 13, 22\%, 24\%, 25\%, 26\%, 28\% and $29 \%$ porosities of the core are also considered for checking the variations of relative sensitivity for the $3.5 \mu \mathrm{m}$ absorption wavelength of $\mathrm{CH}_{4}$ gas at $3.0 \mu \mathrm{m}$ pitch and 0.7 air filling fraction of the cladding. It is observed from Figure 13 that maximum porosity of $29 \%$ offers the maximum relative sensitivity of $21.2 \%$ and minimum porosity of $22 \%$ offers minimum relative sensitivity of $16.7 \%$. This result is also reasonable because maximum porosity of the core contains 


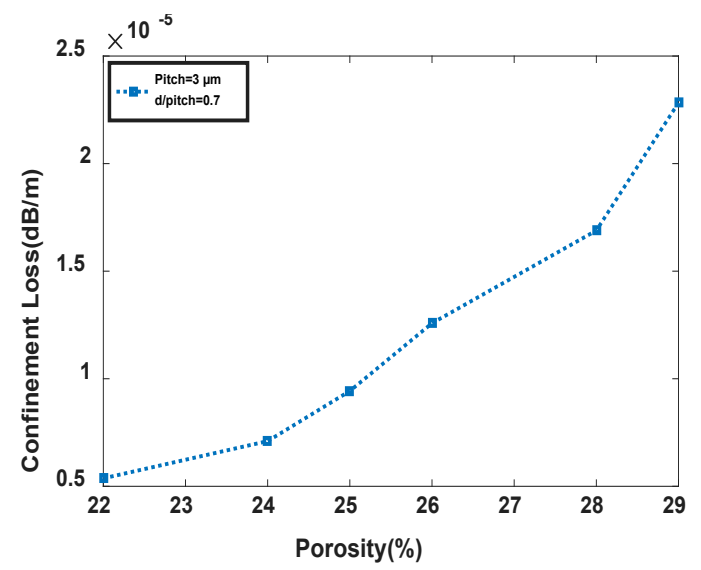

Figure 12. Confinement loss vs. porosity curve at pitch $=3.0 \mu \mathrm{m}, \mathrm{d} / \mathrm{pitch}=0.7 \mathrm{and}$ absorption wavelength of $\mathrm{CH}_{4}$ gas $\lambda=3.5 \mu \mathrm{m}$ of HPC-PCF.

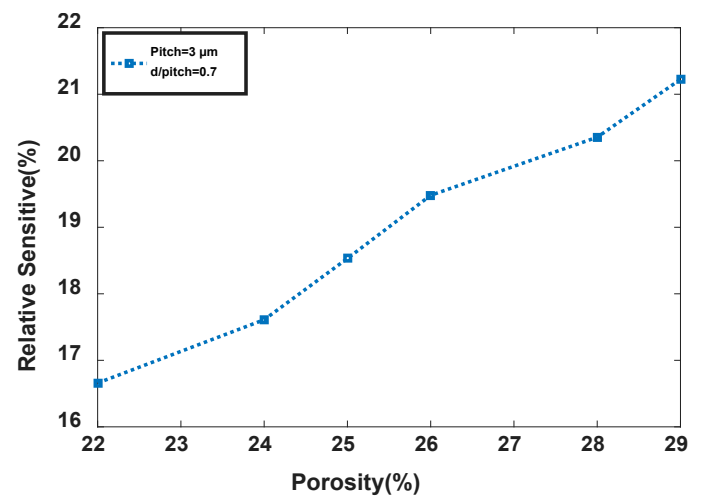

Figure 13. Relative sensitivity vs. porosity curve at pitch $=3.0 \mu \mathrm{m}, \mathrm{d} / \mathrm{pitch}=0.7 \mathrm{and}$ absorption wavelength of $\mathrm{CH}_{4}$ gas $\lambda=3.5 \mu \mathrm{m}$ of HPC-PCF.

maximum amount of gas. In that case, more interaction happens between gas elements and the light for the same absorption wavelength of $\mathrm{CH}_{4}$ gas. So in Figure 13, maximum porosity of the core provides maximum relative sensitivity than minimum porosity for the same absorption wavelength of $\mathrm{CH}_{4}$ gas of HPC-PCF.

\section{Conclusion}

We have proposed and analyzed the $\mathrm{CH}_{4}$ gas sensor based on octagonal cladding with circular air holes and hexagonal core with rectangular and circular air holes of HPC-PCF. The whole design and simulation work of HPC-PCF have been accomplished by COMSOL Multiphysics software through using full vectorial FEM. In this design of HPC-PCF, $2.80 \mu \mathrm{m}, 2.85 \mu \mathrm{m}, 2.90 \mu \mathrm{m}$ and $3.0 \mu \mathrm{m}$ pitches of the cladding and $22 \%, 24 \%, 25 \%, 26 \%, 28 \%$ and $29 \%$ porosities of the core are considered for optimizing the HPC-PCF. In the end, we have achieved optimum confinement loss of $0.000025 \mathrm{~dB} / \mathrm{m}$ and relative sensitivity of $21.2 \%$ for $3.0 \mu \mathrm{m}$ pitch, 0.7 air filling ratio of the cladding and $29 \%$ porosity of the core at $3.5 \mu \mathrm{m}$ absorption wavelength of $\mathrm{CH}_{4}$ gas. In addition, we have determined the numerical 
value of effective refractive index, effective area, nonlinearity and numerical aperture of $1.3725,52.5 \mu \mathrm{m}^{2}, 0.9 \mathrm{~W}^{-1} \cdot \mathrm{m}^{-1}$, and 0.26 respectively at $3.5 \mu \mathrm{m}$ absorption wavelength of $\mathrm{CH}_{4}$ gas. Note that $\mathrm{CH}_{4}$ gas is used in various commercial purposes like generation of electricity, domestic heating and cooking, as an ideal fuel for some modern vehicles and also other applications. Moreover, presence of $\mathrm{CH}_{4}$ gas in all the places is not secured for the human beings because it may create serious accident like fire. So it is more essential to detect the presence of $\mathrm{CH}_{4}$ gas correctly. The proposed design of HPC-PCF will keep optimistic and innovative role for detecting $\mathrm{CH}_{4}$ gas properly which will supportive for global economic in future.

\section{Conflicts of Interest}

The authors declare no conflicts of interest regarding the publication of this paper.

\section{References}

[1] Lu, J., Yu, C., Chang, H., Chen, H., Li, Y., Pan, C. and Sun, C. (2008) Terahertz Air-Core Microstructure Fiber. Applied Physics Letters, 92, 064105. https://doi.org/10.1063/1.2839576

[2] Morshed, M., Hassan, M.I., Roy, T.K., Uddin, M.S. and Razzak, S.A. (2015) Microstructure Core Photonic Crystal Fiber for Gas Sensing Applications. Applied Optics, 54, 8637-8643. https://doi.org/10.1364/AO.54.008637

[3] Atakaramians, S., Shahraam Afshar, V., Ebendorff-Heidepriem, H., Nagel, M., Fischer, B.M., Abbott, D. and Monro, T.M. (2009) THz Porous Fibers: Design, Fabrication and Experimental Characterization. Optics Express, 17, 14053-14062. https://doi.org/10.1364/OE.17.014053

[4] Chowdhury, S., Sen, S., Ahmed, K. and Asaduzzaman, S. (2017) Design of Highly Sensible Porous Shaped Photonic Crystal Fiber with Strong Confinement Field for Optical Sensing. Optik, 142, 541-549. https://doi.org/10.1016/j.ijleo.2017.03.123

[5] Olyaee, S. and Naraghi, A. (2013) Design and Optimization of Index-Guiding Photonic Crystal Fiber Gas Sensor. Photonic Sensors, 3, 131-136.

[6] Benabid, F., Knight, J.C., Antonopoulos, G. and St. J. Russell, P. (2002) Stimulated Raman Scattering in Hydrogen-Filled Hollow-Core Photonic Crystal Fiber. Science, 298, 399-402. https://doi.org/10.1126/science.1076408

[7] Couny, F., Benabid, F. and Light, P.S. (2006) Large-Pitch Kagome-Structured Hollow-Core Photonic Crystal Fiber. Optics Letters, 31, 3574-3576. https://doi.org/10.1364/OL.31.003574

[8] Hoo, Y.L., Jin, W., Ju, J. and Ho, H.L. (2009) Numerical Investigation of a Depressed-Index Core Photonic Crystal Fiber for Gas Sensing. Sensors and Actuators B: Chemical, 139, 460-465. https://doi.org/10.1016/j.snb.2009.02.070

[9] Zhang, L., Ren, G. and Yao, J. (2013) A New Photonic Crystal Fiber Gas Sensor Based on Evanescent Wave in Terahertz Wave Band: In Terahertzwave Band: Design and Simulation. Optoelectronics Letters, 9, 438-440.

[10] Tan, Z., Li, X., Chen, Y. and Fan, P. (2013) Improving the Sensitivity of Fiber Surface Plasmon Resonance Sensor by Filling Liquid in a Hollow Core Photonic Crystal Fiber. Plasmonics, 9, 167-173. 
[11] Park, J., Lee, S., Kim, S. and Oh, K. (2011) Enhancement of Chemical Sensing Capability in a Photonic Crystal Fiber with a Hollow High Index Ring Defect at the Center. Optics Express, 19, 1921-1929. https://doi.org/10.1364/OE.19.001921

[12] Woyessa, G., Fasano, A., Markos, C., Stefani, A., Rasmussen, H.K. and Bang, O. (2017) Zeonex Microstructured Polymer Optical Fiber: Fabrifabrication Friendly Fibers for High Temperature and Humidity Insensitive Bragg Grating Sensing. Optical Materials Express, 7, 286-295. https://doi.org/10.1364/OME.7.000286

[13] Pinto, A.M. and Lopez-Amo, M. (2012) Photonic Crystal Fibers for Sensing Applications. Journal of Sensors, 2012, Article ID: 598178. https://doi.org/10.1155/2012/598178

[14] Rahman, A., Rahman, A.K. and Rao, B. (2013) Early Detection of Skin Cancer via Terahertz Spectral Profiling and 3D Imaging. Biosensors \& Bioelectronics, 82, 64-70.

[15] Drukteinis, J.S., Mooney, B.P., Flowers, C.I. and Gatenby, R.A. (2013) Beyond Mammography: New Frontiers in Breast Cancer Screening. The American Journal of Medicine, 126, 472-479. https://doi.org/10.1016/j.amjmed.2012.11.025

[16] Yu, C., Fan, S., Sun, Y. and Pickwell-MacPherson, E. (2012) The Potential of Terahertz Imaging for Cancer Diagnosis: A Review of Investigations Todate. Quantitative Imaging in Medicine and Surgery, 2, 33-45.

[17] Markelz, A.G., Roitberg, A. and Heilweil, E.J. (2000) Pulsed Terahertz Spectroscopy of DNA Bovine Serum Albumin and Collagen between 0.1 and $2.0 \mathrm{THz}$. Chemical Physics Letters, 320, 42-48. https://doi.org/10.1016/S0009-2614(00)00227-X

[18] Fischer, B.M., et al. (2005) Terahertz Time-Domain Spectroscopy and Imaging of Artificial RNA. Optics Express, 13, 5205-5215. https://doi.org/10.1364/OPEX.13.005205

[19] Markelz, A.G., Knab, J.R., Chen, J.Y. and He, Y. (2007) Protein Dynamical Transition in Terahertz Dielectric Response. Chemical Physics Letters, 442, 413-417. https://doi.org/10.1016/j.cplett.2007.05.080

[20] Olyaee, S., Naraghi, A. and Ahmadi, V. (2014) High Sensitivity Evanescent-Field Gas Sensor Based on Modified Photonic Crystal Fiber for Gas Concondensate and Air Pollution Monitoring. International Journal for Light and Electron Optics, 125, 596-600.

[21] Ademgil, H. and Haxha, S. (2015) Highly PCF Based Sensor with High Sensitivity, High Birefringence and Low Confinement Losses for Liquidanalyte Sensing Applications. Sensors, 15, 31833-31842. https://doi.org/10.3390/s151229891

[22] Hassani, A., Dupuis, A. and Skorobogatiy, M. (2008) Low Loss Porous Terahertz Fibers Containing Multiple Subwavelength Holes. Applied Physics Letters, 92, 071101. https://doi.org/10.1063/1.2840164

[23] Sultana, J., Islam, M.S., Atai, J., Islam, M.R. and Abbott, D. (2017) Near Zero Dispersion Flattened, Low-Loss Porous-Core Waveguide Design for Terahertz Signal Transmission. Optical Engineering, 56, Article ID: 076114. https://doi.org/10.1117/1.OE.56.7.076114

[24] Ma, P., Song, N., Jin, J., Song, J. and Xu, X. (2012) Birefringence Sensitivity to Temperature of Polarization Maintaining Photonic Crystal Fibers. Optics \& Laser Technology, 44, 1829-1833. https://doi.org/10.1016/j.optlastec.2011.12.053

[25] Olyaee, S. and Taghipour, F. (2012) Doped-Core Octagonal Photonic Crystal Fiber (O-PCF) with Ultra-Flattened Nearly Zero Dispersion and Low Confinement Loss in a Wide Wavelength Range. Fiber and Integrated Optics, 31, 178-185.

[26] Razzak, S.M.A., Namihira, Y., Begum, F., Kaijge, S., Hai, N.H. and Zou, N. (2007) 
Design of a Decagonal Photonic Crystal Fiber for Ultra-Flattened Chromatic Dispersion. IEICE Transactions on Electronics, E90-C, 2141-2145.

https://doi.org/10.1093/ietele/e90-c.11.2141

[27] Asaduzzaman, S. and Ahmed, K. (2016) Proposal of a Gas Sensor with high Sensitivity, Birefringence and Nonlinearity for Air Pollution Monitoring. Sensing and Bio-Sensing Research, 10, 20-26.

[28] Razzak, S.M.A., Namihira, Y., Begum, F., Kaijage, S., Kinjo, T., Nakahodo, J., Miyagi, K. and Zou, N. (2007) Decagonal Photonic Crystal Fibers with Ultra-Flattened Chromatic Dispersion and Low Confinement Loss. Optical Fiber Communication Conferencel National Fiber Optic Engineers Conference (OFC/NFOEC), Anaheim, 25-29 March 2007, 1-6.

[29] Ademgil, H. (2014) Highly Sensitive Octagonal Photonic Crystal Fiber Based Sensor. Optik: International Journal for Light and Electron Optics, 20, 6274-6278.

[30] Hao, R., Li, Z., Sun, G., Niu, L. and Sun, Y. (2013) Analysis on Photonic Crystal Fibers with Circular Air Holes in Elliptical Configuration. Optical Fiber Technology, 19, 363-368. https://doi.org/10.1016/j.yofte.2013.04.005

[31] Hou, Y., Fan, F., Jiang, Z.-W., Wang, X.-H. and Chang, S.-J. (2013) Highly Brief Ringent Polymer Terahertz Fiber with Honey Comb Cladding. Optik: International Journal for Light and Electron Optics, 124, 3095-3098.

https://doi.org/10.1016/j.ijleo.2012.09.040

[32] http://www.google.com/search?q=Refractive+index+of+gases\&sa 